\title{
Effect of slotted exit orifice on performance of plasma synthetic jet actuator
}

\author{
Haohua Zong ${ }^{1}$ Marios Kotsonis ${ }^{1}$
}

Received: 20 July 2016 / Revised: 6 December 2016 / Accepted: 14 December 2016 / Published online: 16 February 2017

(C) The Author(s) 2017. This article is published with open access at Springerlink.com

\begin{abstract}
This study experimentally investigates the influence of exit orifice shape on the performance characteristics of a three-electrode plasma synthetic jet actuator. High-speed Schlieren imaging system and phase-locked two-component PIV measurements are used for flowfield characterisation in quiescent conditions. Two actuator configurations with the same exit area but different exit orifice shape (round orifice and slot orifice) are studied. Results indicate a close correspondence between the shapes of the starting vortex ring with the shapes of the respective exit orifices. For the slot orifice, the elongated starting vortex ring gradually expands during propagation, while its ends become warped. A distinct $\mathrm{K}-\mathrm{H}$ instability structure is observed, inducing continuous oscillation of the high-speed jet. Compared with the jet from the round orifice, the slot jet has a higher entrainment rate of surrounding air, thus resulting in a lower propagation velocity of the jet front. The exit velocity of PSJA within one period initially shows a rapid increase, then persists at a relatively high level $(100-130 \mathrm{~m} / \mathrm{s})$, and finally drops with some small-scale oscillations. The oscillation amplitude is less than $10 \mathrm{~m} / \mathrm{s}$, and the oscillation period is approximately $600 \mu \mathrm{s}$. Under conditions of same exit area, orifice shape has little influence on the variation of the exit velocity.
\end{abstract}

Haohua Zong

h.zong-1@tudelft.nl

1 Faculty of Aerospace Engineering, Delft University of Technology, 2629 Delft, HS, The Netherlands

\section{Introduction}

Active flow control techniques including pulsed jets, suction, synthetic jets, and plasma-based actuation are studied extensively (Cattafesta and Sheplak 2011). Compared with other methods, plasma-based actuators are characterised by extremely fast response, wide-bandwidth and very simple and robust construction. These features attracted considerable attention from the flow control community in the last 15 years (Corke et al. 2010; Li et al. 2012; Bletzinger et al. 2005; Popkin et al. 2013a, b).

Dielectric barrier discharge actuators (DBDA), arc discharge actuators (ADA) and plasma synthetic jet actuators (PSJA) are three typical types of plasma actuators. DBDA are characterised by low energy consumption $(O(\mathrm{~W}))$, but the application is so far confined to low-speed flows, owing to the weak induced velocity (typically less than $10 \mathrm{~m} / \mathrm{s}$ ) (Corke et al. 2010; Li et al. 2012; Forte et al. 2007). ADA mainly utilise arc heating effect to interact with high-speed flows. Although some promising results were obtained towards shock wave manipulation and jet noise mitigation, the power consumption $(O(\mathrm{~kW}))$ has been prohibitive for practical applications (Utkin et al. 2007; Wang et al. 2009). By converting the heating energy of pulsed arc to gas mechanical energy, PSJA successfully realises the production of high-speed ( $>400 \mathrm{~m} / \mathrm{s}$ ) high-frequency $(>5 \mathrm{kHz})$ jets, while utilising an acceptable power consumption $(0$ $(100 \mathrm{~W}))$ (Grossman et al. 2003; Narayanaswamy et al. 2010; Zong et al. 2015a, b; Wang et al. 2014). Several evaluation studies on the control authority of PSJA have been performed, such as shock wave manipulation, jet noise mitigation, shock wave boundary layer interaction control and aerofoil trailing edge separation control (Narayanaswamy et al. 2012; Emerick et al. 2014; Caruana et al. 2009, 2013; 
Hardy et al. 2010; Anderson and Knight 2012; Greene et al. 2015).

A major outcome of the previous studies is that the performance of PSJA is not sufficient for high Reynolds number flows. This is because the control effect is shortlived and only confined in the near region of exit orifices (Emerick et al. 2014; Anderson and Knight 2012). In order to further improve the control authority, the influence of actuation parameters on the characteristics of PSJA should be investigated systematically including the geometrical configuration of the PSJA and the architecture of the used power supplies.

For the power supply, different approaches result in various discharge types, deposited energy levels and discharge timescales. Following an increasing energy level criterion, commonly used discharge types can be classified into nanosecond-pulsed discharge, pulsed DC discharge and capacitive discharge (Zong et al. 2015; Zhu et al. 2015; Shin 2010; Reedy et al. 2013; Belinger et al. 2011). Nanosecond-pulsed discharge merits from a high heating efficiency ( $>40 \%$ ), however can only be used to feed small-volume PSJA (tens of $\mathrm{mm}^{3}$ ), due to the low discharge energy available from standard nanosecond pulses $(<20 \mathrm{~mJ}$ ) (Zhu et al. 2015; Xu et al. 2014). When pulsed DC discharge is exploited to accommodate the PSJA, both the discharge current and the discharge time can be tuned independently, whereas the majority of discharge energy is converted into the molecular vibrational energy, leading to a low heating efficiency ( 10\%) (Narayanaswamy et al. 2010; Shin 2010; Reedy et al. 2013). Additionally, at high frequency $(10 \mathrm{kHz})$, misfires are commonly observed due to the limited refresh time (Narayanaswamy et al. 2010). Finally, for capacitive discharge type, the discharge efficiency can reach as high as 90\% (Wang et al. 2014a, b; Belinger et al. 2014). As the stored capacitor energy increases, the jet velocity, jet duration time and pulsed thrust also increase, while the heating efficiency decreases (Golbabaei-Asl et al. $2013,2015)$. With capacitor energy of $4 \mathrm{~J}$, a peak jet velocity of $500 \mathrm{~m} / \mathrm{s}$ can be attained (Reedy et al. 2013).

Geometrical features of PSJA include cavity parameters [volume, aspect ratio (diameter/height)], electrode parameters (distance, location, and shape), and exit orifice parameters (exit diameter, throat length). When the deposited energy is fixed, an increase in the cavity volume results in a decrease in the peak jet velocity and pulse thrust (Cybyk et al. 2003). Increasing inter-electrode distance results in an enlarged heating volume and a significant improvement of jet strength (Zong et al. 2016). This variation is directly related to the mechanical energy dissipation due to the arcinduced shock waves. When the discharge location moves from the cavity bottom towards the actuator exit, jet duration time decreases but strong temporal oscillations of the exit velocity are observed, possibly due to the enhanced shock wave reflections (Zhang et al. 2015). Electrode shape can affect the impedance matching of the discharge circuit, and a large length-to-diameter ratio of electrodes results in a high cut-off working frequency (Popkin et al. 2013b). As the exit diameter increases, an increase of the peak jet velocity is observed while the jet duration time drops. Additionally, if the deposited energy is kept constant, the saturation frequency, which corresponds to the maximum jet mechanical energy, increases linearly with the exit diameter (Zong et al. 2015). Throat length has no influence on the single-pulse performance of PSJA (peak jet velocity, pulsed thrust etc.), but a long throat length has detrimental effects on the repetitive working performance of PSJA at high frequency (Zhang et al. 2015).

Overall, several studies have provided the description of the effect of several geometrical and electrical parameters on the performance of PSJA. In contrast, the orifice shape of PSJA has received no attention to this point, although its effect on the evolving jet can be significant. The influence of exit orifice shapes on the performance of traditional, non-plasma-based synthetic jets has been researched extensively in the literature (Hashiehbaf and Romano 2013; Ho and Gutmark 1987; Dhanak and Bernardinis 1981; Glezer and Amitay 2002; Krothapalli et al. 1981; Grinstein 1995). Results show that a noncircular exit jet has a much higher entrainment rate than a circular jet with the same exit area (Hashiehbaf and Romano 2013; Ho and Gutmark 1987). Additionally, some complex phenomena including azimuthal distortion, break-up and axis switching are present in the self-induced motion of vortex rings issued from slot orifices (Dhanak and Bernardinis 1981; Grinstein 1995). This distinction in mixing and vortex evolutions can significantly change a given outer flow, such as the velocity profiles of boundary layer and its ability to resist an adverse gradient flow (Caruana et al. 2009, 2013; Greene et al. 2015). Hence, the influence of orifice shape on flow field of PSJA needs to be identified towards improving control authority. In the present study, two actuator configurations with different exit orifice shapes (round orifice and slot orifice) but same exit area are characterised. Highspeed Schlieren imaging and phase-locked PIV systems are adopted to investigate the influence of orifice shape on performance characteristics of PSJA.

\section{Experimental setup}

\subsection{Actuator and power supply}

A three-electrode PSJA is constructed for the purposes of this study, mainly comprised of a ceramic cavity and a metallic lid, as shown in Fig. 1. The cavity structure, made of glass ceramic (MACOR), is $15 \mathrm{~mm}$ in height and $12 \mathrm{~mm}$ 


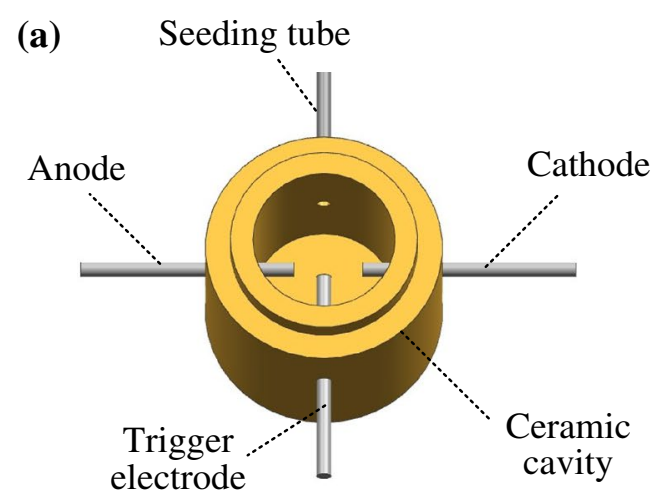

(b)

Round orifice $\quad$ Slot orifice

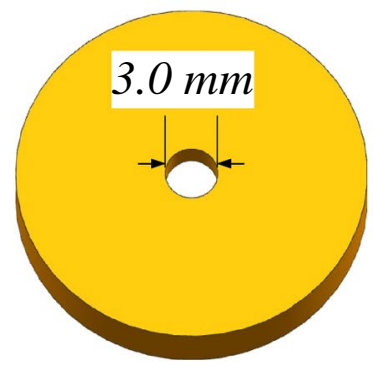

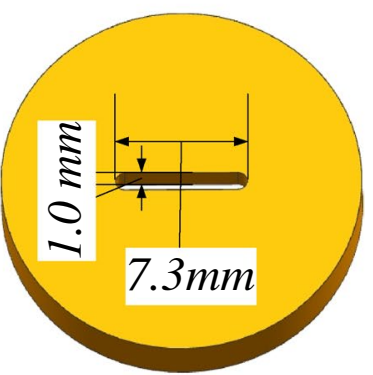

Fig. 1 Actuator components. a Ceramic cavity. b Metal lids for round and slotted exit orifice

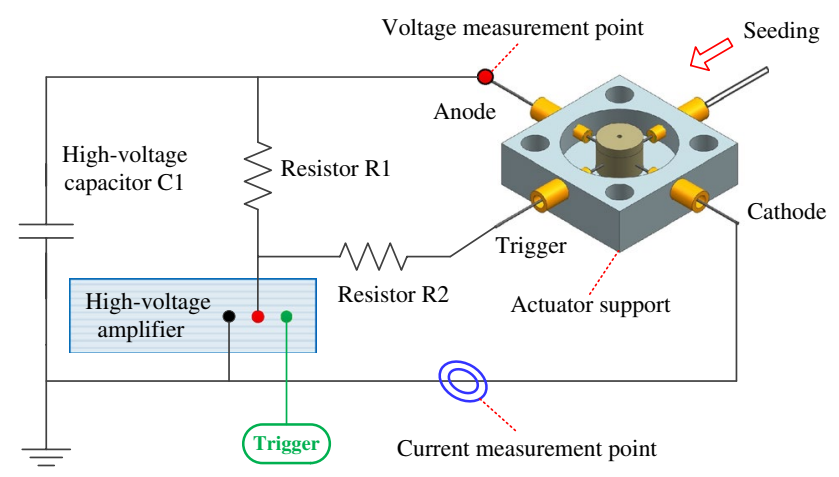

Fig. 2 Power supply system

in diameter. Four holes of a diameter of $1.2 \mathrm{~mm}$ are drilled at equidistant azimuthal locations in the plane lying at 7.5$\mathrm{mm}$ distance from the cavity bottom. Three of the available holes accommodate tungsten needles, which serve as the anode, cathode and trigger electrode, respectively. The remaining hole is connected with a metal capillary tube (inner diameter $0.6 \mathrm{~mm}$ ), serving as a supply port for seeding particles into the cavity during PIV measurement. Distance from the anode, cathode and trigger electrode to the actuator axis is fixed as 1,2 and $0.5 \mathrm{~mm}$, respectively. Two metallic lids with different orifice shapes but same exit area are machined, as shown in Fig. 1b. The first lid features a conventional circular exit orifice with a diameter of $3 \mathrm{~mm}$. The second lid carries a rounded slot orifice, with a length of $7.3 \mathrm{~mm}$ and width of $1 \mathrm{~mm}$ resulting in an aspect ratio (AR: length/width) of 7.3. The throat length for the two orifices is $2 \mathrm{~mm}$.

The adopted power supply system is constructed using a high-voltage amplifier (Trek Model 20/20 C) and several electrical components, as shown in Fig. 2. Compared with the power supply system previously used in Zong et al. (2015a, b), this power supply system is similar in working principle (trigger discharge-capacitive discharge) but simpler in construction (no DC power supply is involved).
For the activation of the discharge sequence, both triggering and charging functions are realised by the highvoltage amplifier. Initially, a $2.5 \mathrm{kV}$ pulse (pulse width: $1600 \mathrm{~ms}$ ) is provided, to charge the high-voltage capacitor $\mathrm{C} 1(1 \mu \mathrm{F})$ while the resistor $\mathrm{R} 1(200 \mathrm{k} \Omega, 100 \mathrm{~W})$ is limiting the charging current in order to protect the HV amplifier. Shortly after the charging process terminates, an $8 \mathrm{kV}$ highvoltage pulse (pulse width: $100 \mu \mathrm{s}$ ) is produced in order to ignite the discharge channel. Once air breakdown is established, a low-resistance channel will be formed between the cathode and the anode, facilitating the rapid release of the energy stored in capacitor C1. Resistor R2 is used to prevent the large capacitive discharge current from flowing back to the amplifier.

\subsection{Measurement system}

High-speed Schlieren imaging and phase-locked Particle Image Velocimetry (PIV) are exploited to visualise the induced flow field of the PSJA. A typical Z-type Schlieren system, consisting of a light source, two concave mirrors, and a high-speed camera (PCO Dimax S4, 12 bit, 4MPix), is constructed on a self-balancing optical table. A continuous light source (Euromex Illuminator, EK-1) in conjunction with an adjustable circular iris provides illumination to the Schlieren arrangement. The light beam radiated from the point light source is first reflected by two concave mirrors (diameter: $30 \mathrm{~cm}$, focal length: $3 \mathrm{~m}$ ), subsequently converged by a convex lens (focal length of $200 \mathrm{~mm}$ ) and finally projected directly on the camera sensor. Between the camera sensor and the convex lens, a knife edge is placed in vertical orientation, facilitating visualisation of density gradients in the direction parallel to the exit plane. 12 bit Schlieren images are recorded at $1.28-\mu \mathrm{s}$ exposure time and $20 \mathrm{kHz}$ acquisition frequency. The resolution and scaling factor of the images are $240 \times 500$ pixels and $0.0926 \mathrm{~mm} / \mathrm{pixel}$, respectively. After the acquisition, Schlieren images are 
post-processed, using techniques of background subtraction and intensity range normalisation (scaling 0-255), in order to improve the contrast ratio. For each case, 20 repetitions of single-pulse actuation are recorded to obtain the statistically averaged jet front positions.

The planar, two-component PIV system consists of a dual-head Nd: YAG laser (Quantel, CFR PIV-200, 200 mJ/ pulse) and a LaVision camera (Imager Pro LX, resolution: $3248 \times 4872$ ). The laser beam is conditioned using a set of optics and knives into a sheet of approximately $0.6 \mathrm{~mm}$ thickness, illuminating the area in the vicinity of the actuator exit orifice. A field of view of $34 \times 51 \mathrm{~mm}^{2}$ is imaged with a Nikon Micro-Nikkor 200-mm macro lens. The actuator is placed in a close Plexiglas box to ensure quiescent flow conditions. Both the box and the cavity are seeded with dielectric oil-based particles (Shell Ondina, mean diameter: $1 \mu \mathrm{m}$ ), generated by an atomizer (TSI, 9302). The seeding provided to the actuator cavity is controlled by a solenoid valve (FESTO, MHJ10), and kept switched off before the discharge, in order to eliminate the interference on the developing flow field. Davis 8.3 software is used to record and process the particle images. For vector processing, an interrogation window of $32 \times 32$ and an overlap ratio of $75 \%$ is adopted resulting in a final vector spacing of 12 vectors $/ \mathrm{mm}$.

The deterministic time delay between discharge ignition and PIV recording $(t)$ ranges from 100 to $2000 \mu$ s in order to track the evolution of the developing flow field. The time step between two subsequent phases is chosen to be 50 or $100 \mu$ s to facilitate the direct comparison with Schlieren results. For each phase, 200 PIV image pairs are recorded to obtain phase-averaged velocity fields. For the round orifice, only the symmetry plane is chosen as the measurement plane. However, in the case of slot orifice, a total of 5 planes are measured, as shown in Fig. 3. One of the measurement planes is the coordinate plane $x z(y=0 \mathrm{~mm})$. The remaining four planes are aligned in the $y z$ plane, starting from $x=0 \mathrm{~mm}$ and ending at $x=3 \mathrm{~mm}$, with a step of $1 \mathrm{~mm}$.
Additionally to the Schlieren imaging and PIV measurements, electrical measurements are performed towards quantifying the discharge behaviour. The trigger voltage $\left(U_{t}\right)$, discharge voltage $\left(U_{d}\right)$ and discharge current $\left(I_{d}\right)$ are measured by a high-voltage probe (LeCroy, PPE20kV) and a current monitor (Pearson, Model 325), and recorded by a digital oscilloscope (Tektronix, TDS $3054 \mathrm{C}$ ) at $25 \mathrm{MHz}$ sampling rate. Measurement stations are indicated in Fig. 2. Based on the measured discharge voltage and discharge current, the instantaneous discharge power $\left(P_{d}\right)$ can be calculated.

Typical discharge waveforms are shown in Fig. 4. The breakdown instant is set as the origin of time axes. Prior to the breakdown of electrode gap, $U_{t}$ increases almost linearly with time and peaks at about $7 \mathrm{kV}$. During discharge ignition, the capacitor voltage drops sharply from $2.5 \mathrm{kV}$ to approximately $0.1 \mathrm{kV}$. This sharp drop is caused by the change of load characteristics in the discharge channel. Prior to breakdown, the discharge channel can be treated as an infinite resistor and all the capacitor voltage is applied on the gap. During ignition, a spark (arc) is formed and resistance of the discharge channel drops significantly from $\sim \infty$ to 0 (100 m $\Omega$ ) (Laurendeau et al. 2014). Note that the discharge capacitor is not an ideal capacitor. Its parasitic resistance and inductance can be comparable to or even larger than the wire inductance and the arc resistance. Thus, a considerable portion of the initial voltage $(2.5 \mathrm{kV})$ is burdened by the parasitic resistance and inductance of the discharge capacitor. As a result, the voltage drop at the arc, namely the measured discharge voltage after ignition, is very small $(0(0.1 \mathrm{kV}))$.

After discharge initialisation, the capacitor voltage experiences a quasi-periodical oscillation (ringing) with a period of about $75 \mu \mathrm{s}$. During the oscillation, the voltage envelope amplitude gradually decreases. Based on the measured voltage and current signals, the duration of the discharge is estimated to be approximately $260 \mu \mathrm{s}$. The variation of the discharge current is similar to the arc voltage, and the observed peak discharge current is $183 \mathrm{~A}$. This
Fig. 3 PIV measurement planes. a Slot orifice; $\mathbf{b}$ round orifice

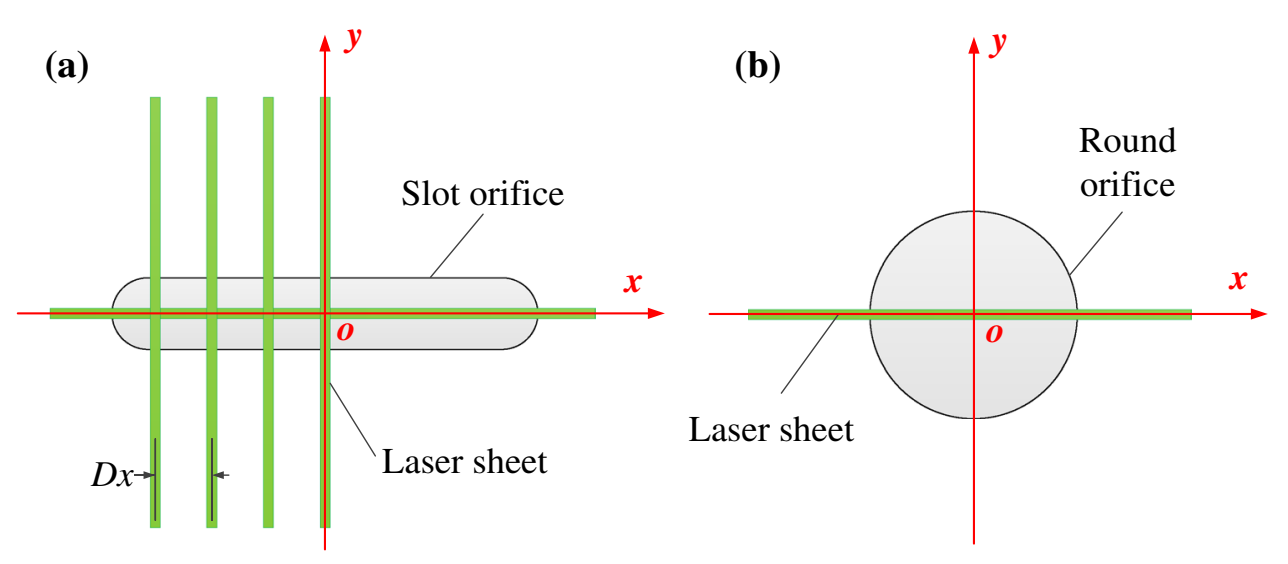



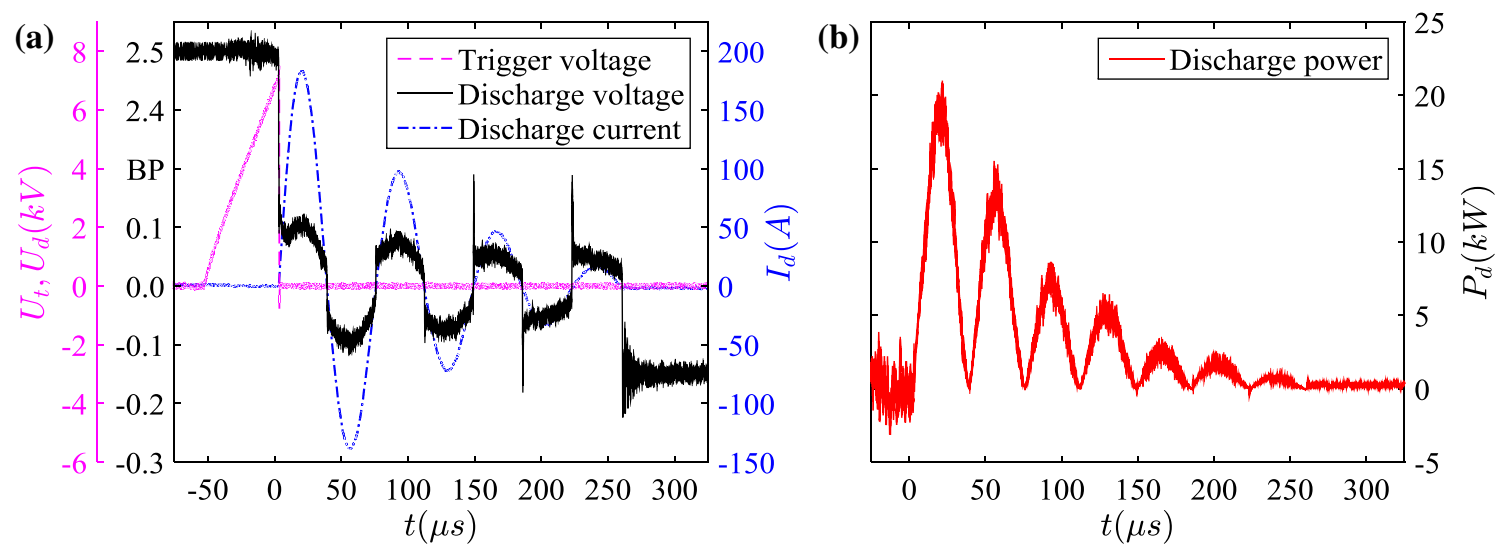

Fig. 4 Discharge waveforms. a Trigger voltage, discharge voltage and discharge current; note that the vertical axis for applied voltage has a break point (BP) and the scale jumps from 0.2 to $2.3 \mathrm{kV}$. b Instantaneous discharge power

periodical oscillation behaviour of discharge waveforms indicates that the discharge circuit behaves similarly to a RLC circuit (Belinger et al. 2014). Regarding instantaneous discharge power, 7 pulses with decreasing amplitudes are observed. The power pulse period is estimated to be $38 \mu$ s, half of the oscillation period of the discharge voltage. Moreover, the peak discharge power reaches $20 \mathrm{~kW}$ and no negative values are observed. By integrating the instantaneous discharge power, total discharge energy is estimated to be $1056 \mathrm{~mJ}$.

\section{Experimental results}

In this section, Schlieren imaging snapshots and phaseaveraged PIV measurements are presented sequentially. Based on these results, the evolution characteristics of the starting vortex ring and high-speed jet, emanating from the actuator exit orifice, are analysed. Influence of the orifice shape on major performance parameters of PSJA, including jet front velocity, penetration length and exit velocity are investigated in detail.

\subsection{Schlieren imaging}

\subsubsection{Flow field evolution}

Shortly after initiation of the discharge, a series of weak compression waves (shock waves) are formed. Since the coherence of these flow structures would be compromised in statistically averaged Schlieren images, instantaneous Schlieren images between $t=100 \mu$ s and $t=$ $350 \mu$ s are shown in Fig. 5 for clarity. Instances between $t$ $=400 \mu \mathrm{s}$ and $t=2000 \mu \mathrm{s}$ are shown in Fig. 6. At $t=100$ $\mu$, several shock waves are formed due to the rapid pressure rise in the actuator cavity caused by the capacitive arc discharge. In the case of the slot orifice, the observed shock wave is in a bow shape in the $x z$ plane, slightly different from the half-circle shape of the other shock waves. A clearly identified starting vortex ring is observed at $t=$ $150 \mu$ s. Combining the two Schlieren views in $x z$ plane and $y z$ plane, it becomes evident that the starting vortex ring of the slotted actuator exhibits an elongated shape. This is rather expected, since vorticity distribution of the starting vortex ring is conditioned from the shear layer developing in the throat of the exit orifice. Additionally, some weak shock waves, possibly caused by shock wave reflection in the cavity, can still be seen at $t=150 \mu \mathrm{s}$. For the round orifice, the front vortex ring gradually grows in size, and finally evolves into a spherical vortex (as indicated in the Schlieren image at $t=250 \mu \mathrm{s}$ ), due to rigorous entrainment of surrounding fluid. However, for slot orifice, quite different scenarios are observed in the two measured planes. The jet body in the $x z$ plane shrinks during the propagation, and distance of the developing vortex pair reduces. In the $y z$ plane, the jet body quickly expands and assumes a mushroom shape at $t=250 \mu \mathrm{s}$. Overall, the projections in $x z$ and $y z$ planes suggest a rapid deformation of the starting vortex for the case of the slot orifice.

As the grayscale in Schlieren images reflects the integration of density gradient along the view direction, a bright/ dark region suggests a large density variation. Regardless of orifice shape, between $t=350 \mu \mathrm{s}$ and $t=700 \mu \mathrm{s}$, a distinctive jet is expelled from the cavity, illustrating that the exiting fluid density changes significantly during this period. The initially issued jet typically is of high density close to the ambient value (cold gas), while the low-density jet (hot gas) always comes out at a later time. This agrees well with the simulation results in Dufour et al. (2013). After $t=800 \mu \mathrm{s}$, the slot jet assumes almost the same width in both $x$ direction and $y$ direction, indicating that 


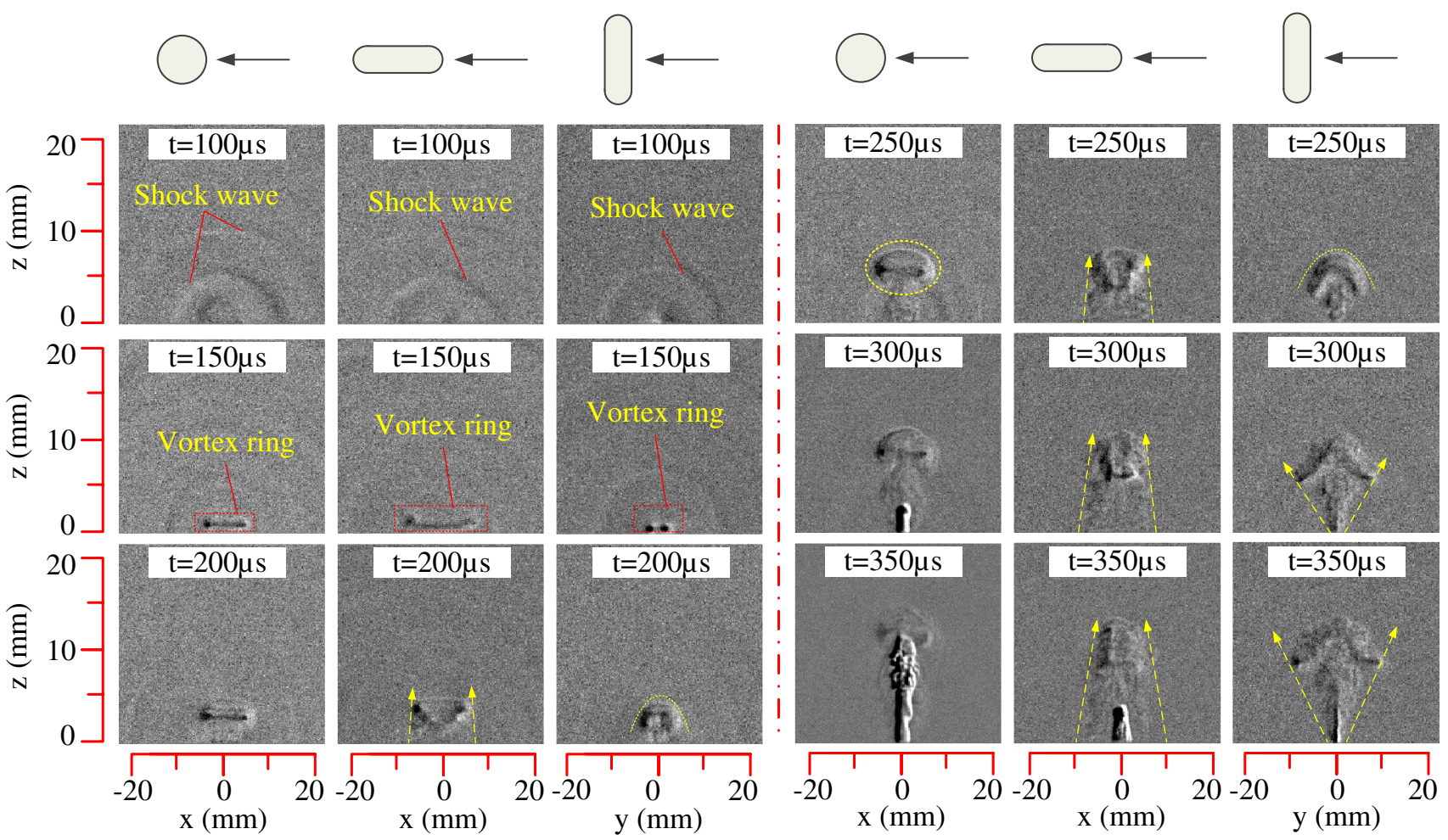

Fig. 5 Instantaneous Schlieren images between $t=100 \mu$ s and $t=350 \mu$ s. Orifice shape and view direction are indicated on the top line

the narrow jet body is gradually evolving into a cylindrical shape (Krothapalli et al. 1981).

\subsubsection{Jet front velocity}

In Fig. 6, the jet front positions are indicated by yellow triangles. It is clear that the slot jet propagates significantly slower than the round jet. This is attributed to viscous drag. More specifically, it is noted that although the exit area between the round and slot orifice is identical, the perimeter of the slot is significantly larger than the perimeter of the round orifice. As such, the contact area between the high-speed core of the jet and the surrounding quiescent fluid is larger for the slot jet, which results in a higher viscous drag and lower propagation speed.

Through accurate detection of the position and trajectory of the jet front, the temporal evolution of the jet front velocity $\left(U_{p}\right)$ can be estimated. Statistically averaged results of 20 pulse repetitions are shown in Fig. 7. The camera sensor detection error is approximately 2 pixels, while the variation of jet front position from shot to shot is typically 3 pixels. Based on these error bounds and the corresponding frame interval $(50 \mu \mathrm{s})$, the uncertainty in velocity calculation can be determined, as indicated by error bars in Fig. 7 . Note that the performance parameters pertaining to the slot orifice shown in Fig. 7 are the average of the two values obtained from the two view directions, respectively.
Within one actuation cycle, the jet front velocity increases, and then decreases. Peak velocity is reached between $t=200 \mu \mathrm{s}$ and $t=250 \mu \mathrm{s}$. For the slot orifice, the peak jet front velocity is slightly lower than that for the round orifice. Note that this peak value is only half of the peak exit velocity as will be shown in the next section.

\subsection{PIV results}

\subsubsection{Uncertainty analysis}

Four sources of velocity estimation errors are identified in the current PIV measurement. The first source of error $\left(\delta u_{1}\right)$ is related to the inherent cross-correlation operation. With a typical particle displacement error of 0.2 pixel and a maximum particle displacement of 10 pixels, $\left(\delta u_{1}\right)$ is estimated to be $2 \% u_{\max }$, where $u_{\max }$ denotes the maximum velocity in flow field. The second source of error is related to the finite ensemble size used for the phase averaging of the measured flow fields. On the premise that observed velocity fluctuations obey a Gaussian distribution can be estimated as follows (Sciacchitano and Wieneke 2016),

$\delta u_{2}=\frac{\sigma_{u}}{\sqrt{N}}$,

$\sigma_{u}$ where is the standard deviation of velocity and $N$ is the sample number (200 in this study). For the investigated 


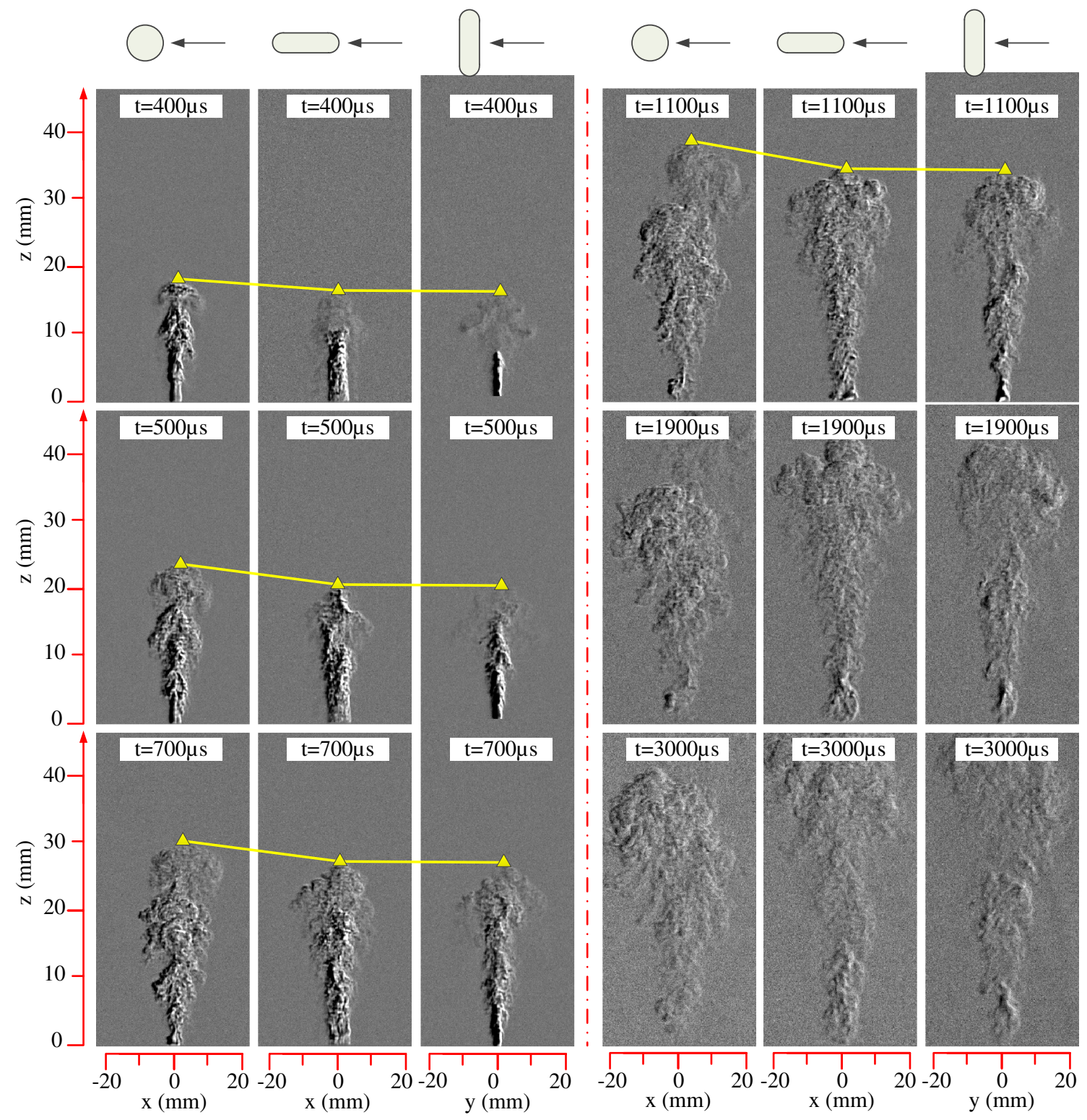

Fig. 6 Instantaneous Schlieren images between $t=400 \mu \mathrm{s}$ and $t=3000 \mu \mathrm{s}$. Orifice shape and view direction are indicated on the top line

case, the peak value of is just above $30 \mathrm{~m} / \mathrm{s}$, leading to a typical value of of less than $2 \mathrm{~m} / \mathrm{s}$.

The third source of error $\left(\delta u_{3}\right)$ is related to the finite laser sheet thickness (denoted as $\delta L$ ). Theoretically, the jet velocity distribution across the laser sheet is nonuniform and has a top-hat shape for the investigated case, as shown in Fig. 8a. Due to the projection in the 2D PIV vector field, the averaging effect will be proportional to the laser sheet thickness. The effect is particularly important in the case of the slot orifice, where the laser sheet thickness $(0.6 \mathrm{~mm})$ is comparable to the length of the minor axis of the slot $(1 \mathrm{~mm})$. Here, the worst case is considered, where the velocity distribution across the laser sheet is assumed to be parabolic. Based on this assumption, the measured velocity $\left(u_{m}\right)$ and the produced estimation error are deduced as follows.

$\left\{\begin{array}{l}u_{m}=\int_{-\delta L / 2}^{\delta L / 2} u(s) d s=\int_{-\delta L / 2}^{\delta L / 2} u(0) \cdot\left(1-\frac{4 s^{2}}{w^{2}}\right) d s \\ \delta u_{3}=u_{m}-u(0)=-\frac{1}{3}\left(\frac{\delta L}{w}\right)^{2} \cdot u(0)\end{array}\right.$

where $u(0)$ is the true velocity; $w$ is the orifice dimension along the coordinate direction (width/length/diameter). As a result, the exit velocity is always underestimated. The estimation error is positively proportional to the ratio of laser sheet thickness to orifice dimension. For the slot 


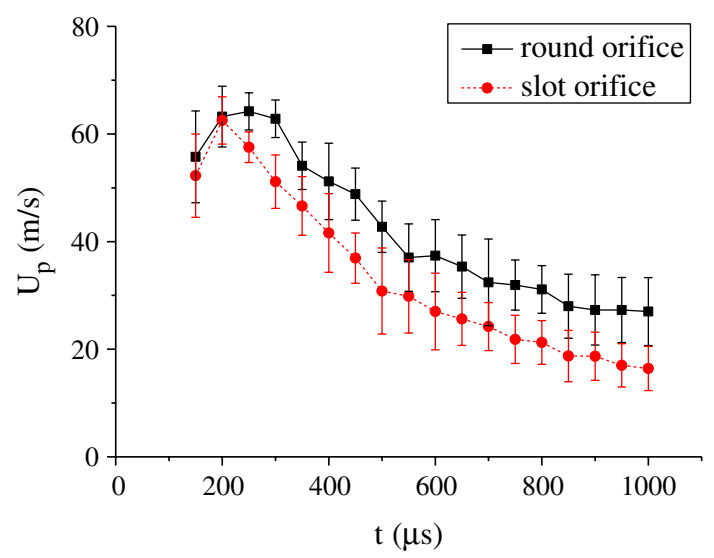

Fig. 7 Temporal evolution of the jet front velocity $\left(U_{p}\right)$ for the two tested actuators

orifice in this study, the peak relative error $\delta u_{3} / u(0)$ in $y=$ $0 \mathrm{~mm}$ measurement plane is approximately $12 \%$. By comparison, the maximum of $\delta u_{3} / u(0)$ for the round orifice is only $1.3 \%$.

The last source of error $\left(\delta u_{4}\right)$ pertains to the discharge timing uncertainty, as illustrated in Fig. $8 \mathrm{~b}$. Based on the obtained electrical signals of the 200 pulse, the discharge timing is demonstrated to be normally distributed, which agrees well with the results in Laurendeau et al. (2015). The standard deviation of the discharge timing $(\delta T)$ is around $2.7 \mu \mathrm{s}$. Under the assumption of a local linear approximation, the relationship between $\delta u_{4}$ and the temporal evolution of velocity [denoted as $u(t)$ ] can be deduced, as shown in Eq. (3). Considering a typical jet acceleration time of $100 \mu$ s and a peak jet velocity of $120 \mathrm{~m} / \mathrm{s}$ (Zong and Kotsonis 2016), the peak value of $\delta u_{4}$ is estimated to be $3.3 \mathrm{~m} / \mathrm{s}$.

$\delta u_{4}=u\left(T_{0}+\delta T\right)-\left.u(T) \approx \delta T \frac{\partial u}{\partial t}\right|_{t=T_{0}}$

The total measurement uncertainty (denoted as) is defined as the Euclidean sum of the above four measurement errors, as follows $\delta u=\sqrt{\delta u_{1}^{2}+\delta u_{2}^{2}+\delta u_{3}^{2}+\delta u_{4}^{2}}$

It should be noted that $\delta u$ is dependent both on space coordinates (location in the measurement domain) and time delay, and as such not defined by a single value. For the remainder of this study, the local measurement uncertainty will be propagated through the performed analysis in order to produce uncertainty bounds for extracted parameters such as penetration length and jet front velocity.

\subsubsection{Phase-averaged velocity field}

The effect of the orifice shape on the phase-averaged velocity fields between $t=100 \mu$ s and $t=200 \mu$ s is shown in Fig. 9. Here, the two-dimensional Euclidean sum of the measured velocity components is denoted as in the $x z$ plane and in the $y z$ plane, where $U_{x}, U_{y}$ and $U_{z}$ and are the components of velocity in the three coordinate directions. Extensive analysis of the phase-averaged velocity fields pertaining to the round orifice shape is available in (Zong and Kotsonis 2016). Thus, the following section concentrates mainly on the slot orifice actuator and the differences of the latter to the round orifice actuator.

At $t=100 \mu \mathrm{s}$, a quasi-2D jet is issued from the slot orifice with a maximum exit velocity of $50 \mathrm{~m} / \mathrm{s}$, which is considerably larger than that of the round orifice actuator for the same delay. For the slot orifice actuator, the jet closely resembles the overall shape of the orifice. No spherical structures corresponding to shock waves are observed in the phase-averaged velocity fields, due to the dispersion of the shock wave location caused by discharge timing uncertainty. For the slot orifice actuator, the vorticity in the shear layer rolls into an elongated vortex ring at $t=150 \mu \mathrm{s}$. As the jet propagates away from the orifice $(t=200 \mu \mathrm{s})$, its major axis (in the $x$ direction) shrinks while the minor axis (in the $y$ direction) augments. This signifies the gradual transition of the elongated jet body into a cylindrical shape. Such behaviour agrees well with observations pertaining to
Fig. 8 Velocity estimation errors caused by a thick laser sheet and $\mathbf{b}$ discharge timing uncertainty (a)

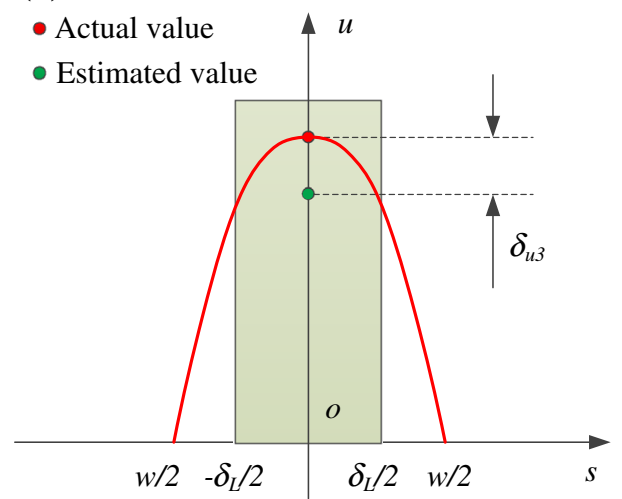

(b)

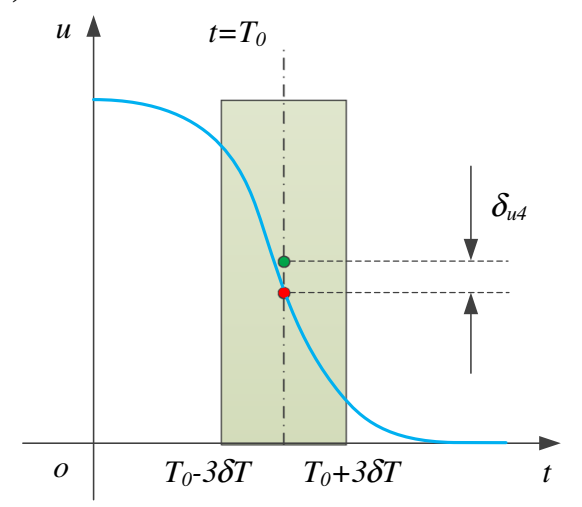




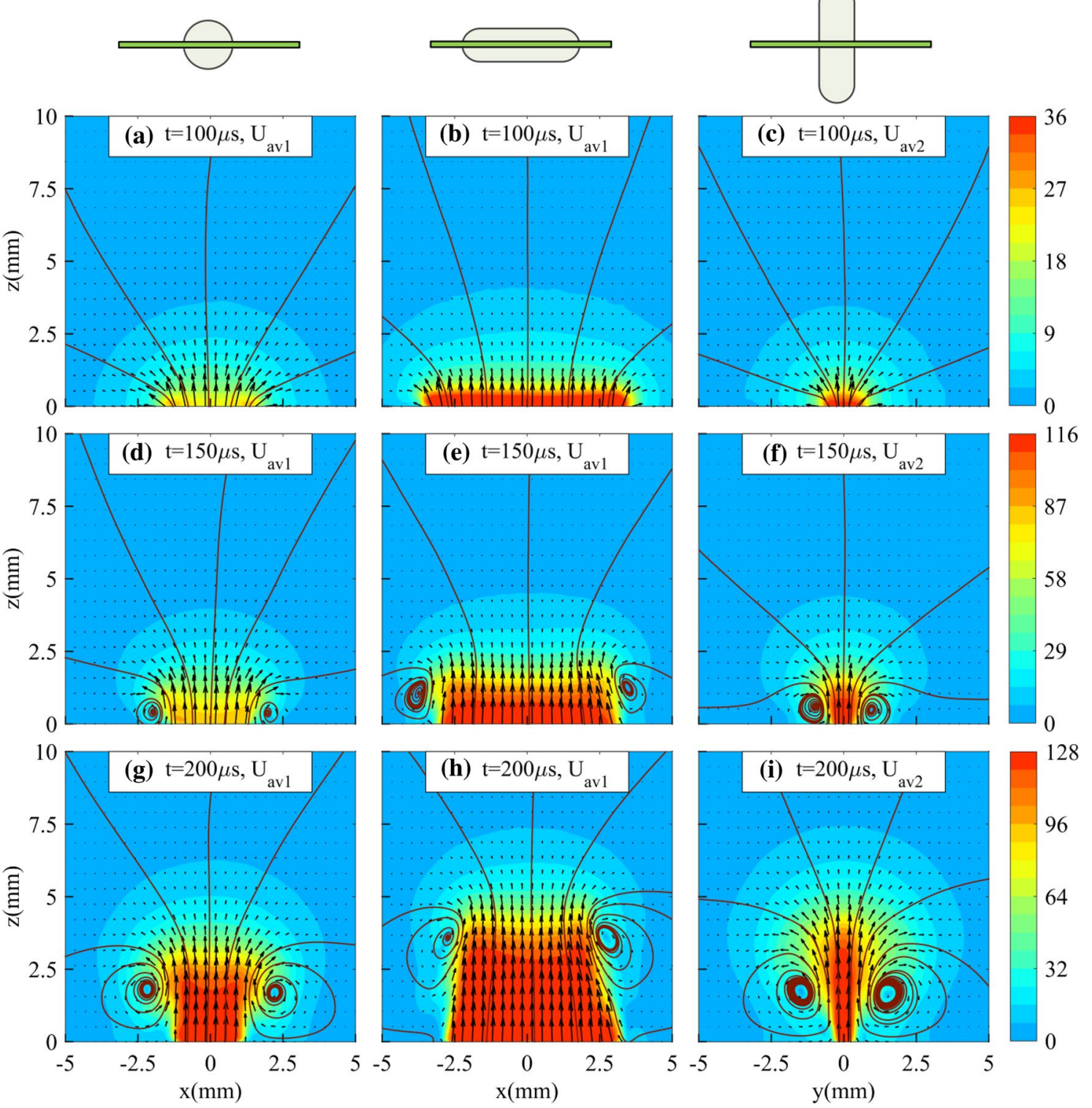

Fig. 9 Phase-lock-averaged velocity field between $t=100 \mu \mathrm{s}$ and $t=200 \mu \mathrm{s}$. Measurement plane and orifice shape are indicated on the top line. The solid lines displayed are streamlines

steady slot orifice jets studied by Dhanak and Bernardinis (1981) and Krothapalli et al. (1981).

Under the assumption that projection of the front vortex ring on the $x y$ plane is an ellipse, a schematic diagram of the vortex ring transformation can be obtained based on the vortex core coordinates extracted from velocity fields. This is shown in Fig. 10. During the propagation of the starting vortex ring, the length of the minor axis gradually increases, and finally exceeds the length of the major axis (at approximately $t=250 \mu \mathrm{s}$ ). The axes-switching behaviour agrees well with the simulation results in Grinstein (1995). In their study, Grinstein et al. conjecture that the fast entrainment rate along the $y$ direction is the principle cause of such axis-switching phenomenon. In addition, the shape of the vortex ring becomes severely warped at the later stages of propagation ( $t>150 \mu \mathrm{s})$. Warping is directly related to the disparate propagation velocity of different parts of the vortex ring. The motion of the front vortex ring is governed by both the momentum input of the highspeed core jet and the viscous drag caused by the shear and entrainment between the jet core and surrounding quiescent fluid. When the vortex ring is initially expelled, it is governed by an almost constant propagation velocity along its perimeter. However, the long edge (along the major axis) of the core jet experiences higher viscous drag than the short edge (along the minor axis) due to larger contact area 
Fig. 10 Front vortex ring characteristics. a Variation of axial length; $\mathbf{b}$ shape transformation during propagation

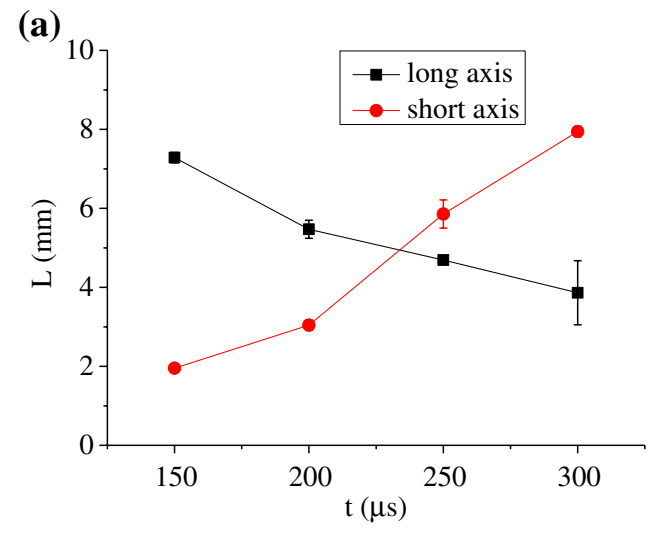

with the quiescent fluid. As a result, the strong shear and the high entrainment rate along the $y$ direction restrict the propagation velocity of the long edge of the front vortex ring, leading to the observed warping.

The phase-averaged velocity fields between $t=300 \mu \mathrm{s}$ and $t=500 \mu$ s are shown in Fig. 11. In Fig. 11c, f, $\mathrm{i}$, the high-speed jet seems to vacillate to the left and right, during the downstream propagation. This is possibly caused by the Kelvin-Helmholtz instability. In order to verify this phenomenon, some post-processing is performed on the velocity field shown in Fig. 11f. First, a threshold value of $70 \mathrm{~m} / \mathrm{s}$ is selected to extract the high-speed jet body from the background, as shown in Fig. 12a. Subsequently, the vector angle of each velocity vector is calculated and averaged in the $y$ direction. Variation of the $y$-averaged jet angle (denoted as $\theta$ ) is shown in Fig. $12 \mathrm{~b}$.

When the jet propagates away from the exit, in the $\mathrm{z}$ direction, the jet angle oscillates in a periodical way, and the oscillation amplitude is rapidly amplified. This agrees well with the basic features of $\mathrm{K}-\mathrm{H}$ instability, where the initial small disturbance is amplified in an exponential way (Lee 1976). This is largely expected since the issued jet can be regarded as a thin shear layer, which provides the environment for $\mathrm{K}-\mathrm{H}$ type instability amplification. Yet, the identification of the source of instability is less straightforward. The evident periodic variation of the jet angle, extracted through phase-averaged data, suggests a strong initial disturbance periodicity. Based on the averaged jet velocity at $t=400 \mu$ s (approximately $70 \mathrm{~m} / \mathrm{s}$ ) and the averaged wavelength in Fig. 12 (approximately $2.6 \mathrm{~mm}$ ), the disturbance period is estimated to be $37 \mu$ s, in close agreement to the period of discharge power (Fig. 4b). This indicates that the initial disturbance frequency and phase is locked to the discharge itself. As stated in Sect. 2.2, the discharge power oscillates periodically, with a decreasing oscillation amplitude. Due to the asymmetric electrode configuration, this periodic energy deposition finally results in the periodic disturbance on the jet exit angle.
Figure 13 presents the phase-averaged velocity fields between $t=600 \mu \mathrm{s}$ and $t=1000 \mu \mathrm{s}$. From $t=400 \mu \mathrm{s}$ to $t$ $=600 \mu \mathrm{s}$, the exit velocity drops sharply from $120 \mathrm{~m} / \mathrm{s}$ to about $40 \mathrm{~m} / \mathrm{s}$. The profile of the high-speed jet attains the form of a tadpole with its head surrounded by the front vortex ring (Fig. 13b-c). At $t=800 \mu$ s, almost no observable jet is issued. However, at $t=1000 \mu$ s, a second jet appears although with a very small velocity $(<10 \mathrm{~m} / \mathrm{s})$. Additionally, the sizes of the front vortex ring and the body of the slot jet show little difference in the $x z$ plane and the $y z$ plane, indicating that the quasi $2 \mathrm{D}$ jet is evolving into an axisymmetric jet.

For ease of visualisation of the topology of the developing jet, the contour surface of $U_{a v 2}$ can be obtained by virtue of a cubic interpolation method, as shown in Fig. 14. Note that the maximum value of $U_{a v 2}$ at $t=800 \mu \mathrm{s}$ is lower than $50 \mathrm{~m} / \mathrm{s}$; thus, the contour surface is changed to $40 \mathrm{~m} / \mathrm{s}$. Although the presented variable is not total velocity, Fig. 14 provides a good visualisation of the evolution of the high-speed jet body. The body of the high-speed jet changes from the initial mushroom shape at $t=300 \mu$ s to a tadpole shape at $t=600 \mu \mathrm{s}$. Finally, it assumes an egg shape once convected sufficiently far from the exit orifice.

\subsubsection{Penetration length and exit velocity}

The previous analysis has shown apparent morphological differences between the two tested cases regarding the evolution of the jet core and the front vortex ring. It is thus instructive to evaluate whether the jet penetration length is also affected by the shape of the exit orifice. It must be noted here that the choice for an independent measure of the penetration length in quiescent conditions is not straightforward due to the absence of reference velocity. As such, a velocity contour line of $10 \mathrm{~m} / \mathrm{s}$ is arbitrarily chosen as the jet edge. The $y$-coordinate of the upmost point of the $10 \mathrm{~m} / \mathrm{s}$ contour line is defined as the penetration length, as shown in Fig. 15. A preliminary sensitivity 

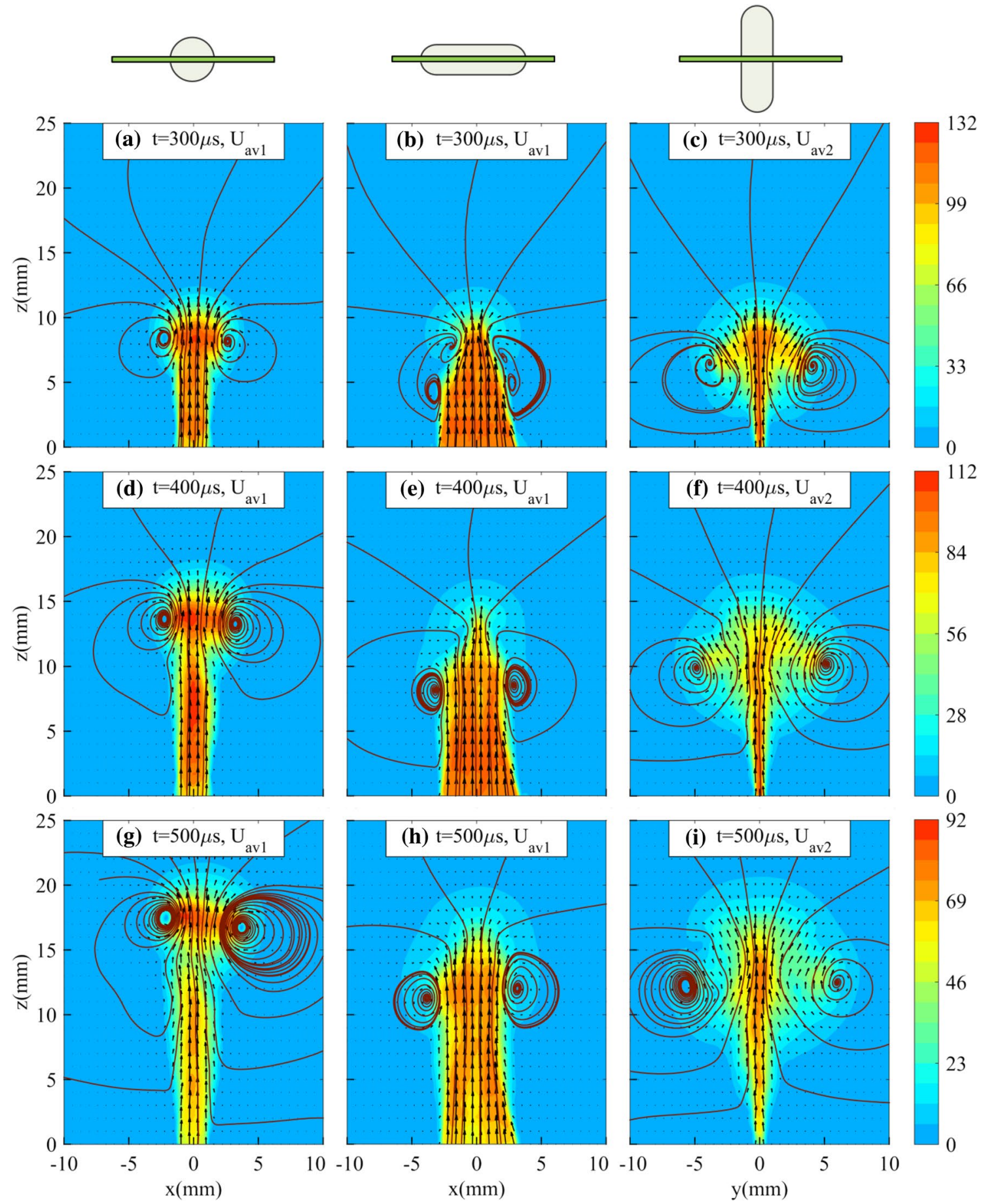

Fig. 11 Phase-lock averaged velocity field between $t=300 \mu$ s and $t=500 \mu$ s. Measurement plane and orifice shape are present in the top line. The solid lines displayed are streamlines

test shows that when the velocity contour line changes from 10 to $5 \mathrm{~m} / \mathrm{s}$, mean variation of the calculated penetration length is less than $2 \mathrm{~mm}$. Due to these considerations, the penetration length presented in this study is largely aimed for comparison between the two actuators and not as a performance metric. The effect of the actuator orifice shape on the estimated penetration length (denoted as $\delta_{p}$ ) is shown in Fig. 16. Additionally, the change rate of the penetration length (denoted as $V_{p}$ ) is also calculated and presented. Note that the values pertaining to the slot orifice shown below in Figs. 16 and 17 are the average of the two values obtained from the two symmetry planes. 
(a)

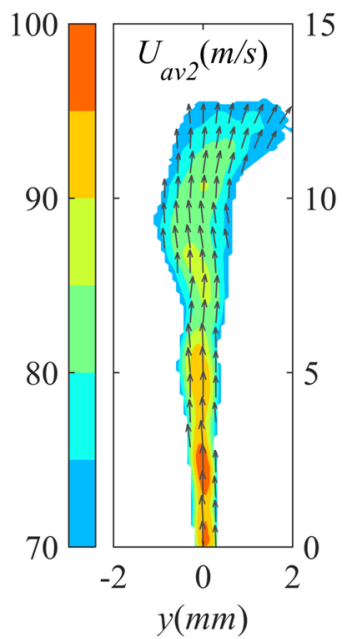

(b)

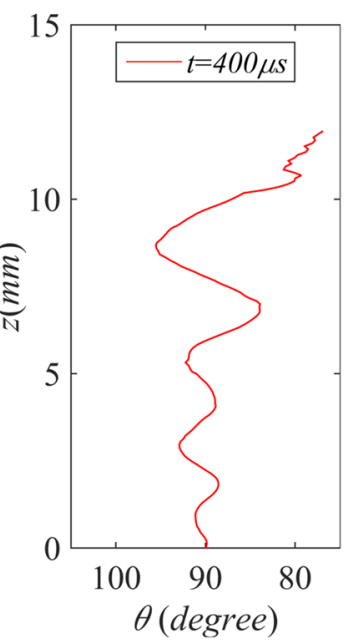

Fig. 12 a Extraction of the high-speed jet body. b Variation of the jet angle

Shortly after discharge ignition, the developing jet propagates and the penetration length gradually increases. Before $t=250 \mu \mathrm{s}$, the change rate of the penetration length for the round orifice is slightly lower than that for the slot orifice. The peak rising rate is around $60 \mathrm{~m} / \mathrm{s}$, close to the peak jet front velocity observed in Fig. 7. Between $t=$ $250 \mu \mathrm{s}$ and $t=900 \mu \mathrm{s}$, the jet emanated from the round orifice penetrates faster than from the slot orifice, which agrees well with the basic trend shown in Fig. 7. This difference in penetration rate can be once again attributed to the jet entrainment/spreading rate, which will be quantified in the next section. Owing to a constant exit area, the slot orifice case has a larger contact surface with the surrounding air, compared to the round orifice case. This leads to a high entrainment rate of low-energy fluids and finally the slow propagation speed of the slot jet. In the case of the round orifice actuator, the penetration length begins to drop after $t=1000 \mu \mathrm{s}$, resulting in a maximum penetration length of $33.8 \mathrm{~mm}$. For the jet pertaining to the slot orifice actuator, the penetration length at $t=1000 \mu \mathrm{s}$ is $29 \mathrm{~mm}$, and no peak point is observed between $t=100 \mu$ s and $t=$ $2000 \mu$ s.

The second indicator of jet intensity can be established based on the exit velocity. In order to obtain the averaged exit velocity at different phases, a rectangle just above the exit is chosen as the monitor region (see Fig. 15). The exit velocity presented in the following discussion is the spatial-averaged velocity in the monitor region. The width of the monitor region is set as half of the slot size, while the height is fixed to $0.5 \mathrm{~mm}$. The variation of the exit velocity in one period for the two tested actuators is shown in Fig. 17.

Prior to $t=200 \mu \mathrm{s}$, an acceleration stage is present, and the exit velocity increases rapidly from about $30 \mathrm{~m} / \mathrm{s}$ to the peak value. Occurrence of the jet acceleration stage is related to the inertia of the throat gas (Zong et al. 2015). Subsequently, between $t=200 \mu$ s and $t=400 \mu$ s, the exit velocity is sustained at relatively high level $(100-130 \mathrm{~m} / \mathrm{s})$ and is approximately double of the peak jet front velocity. This discrepancy can be attributed to the different definitions. The jet front velocity is calculated by tracking the motion of the jet front, which is identified by either the grayscale change in Schlieren images or the velocity contour in PIV results. In contrast, exit velocity is obtained by monitoring the velocity of the gas just expelled from the exit orifice. It can be expected that the gas just expelled will contain higher momentum. During propagation in the $z$ direction, the jet velocity drops rapidly, which leads to a significant difference between the exit velocity and the jet front velocity. It is beneficial to note that the "peak jet velocity" widely determined by Schlieren methods in previous studies (Narayanaswamy et al. 2010; Zong et al. 2015a, b; Wang et al. 2014) severely underestimates the true peak jet velocity, as demonstrated by the PIV measurements of the current study.

After $t=400 \mu \mathrm{s}$, the exit velocity shows a linear decrease, and the first minimum value is reached at $t=800 \mu \mathrm{s}$. Note that this minimum value is still a positive value, suggesting that the jet is still continuing at this time. After that, the jet velocity increases again, reaching a second maximum and a second minimum. It is evident that the emitting jet intensity presents an oscillatory amplitude variation. The oscillation period of the exit velocity is about $600 \mu \mathrm{s}$. The refresh stage finally comes at about $t=$ $1900 \mu$ s, indicated by a small negative exit velocity (fluid entering the actuator). Moreover, the orifice shape appears to have no influence on the exit velocity, which is within expectation. The exit velocity is mainly determined by the dynamic evolution of the cavity gas. With the energy deposition and exit area kept constant, the exit velocity variation should be identical (Zong et al. 2015).

\subsubsection{Jet spreading rate in the time-averaged flow field}

Based on the above analysis, entrainment rate plays a dominant role in the axes-switching phenomenon and the formation of different jet penetration lengths. For steady jets, the entrainment rate can be quantified by the spreading jet profile in time-averaged flow field (Gutmark et al. 2011). The boundary of the jet is typically defined as a fixed fraction of the centreline jet velocity [ $40 \%$ in (Gutmark et al. 2011)]. Extending this approach to the current study, the time-averaged flow field of PSJA (average of all phases) is considered in order to analyse the jet spreading rate. Based 


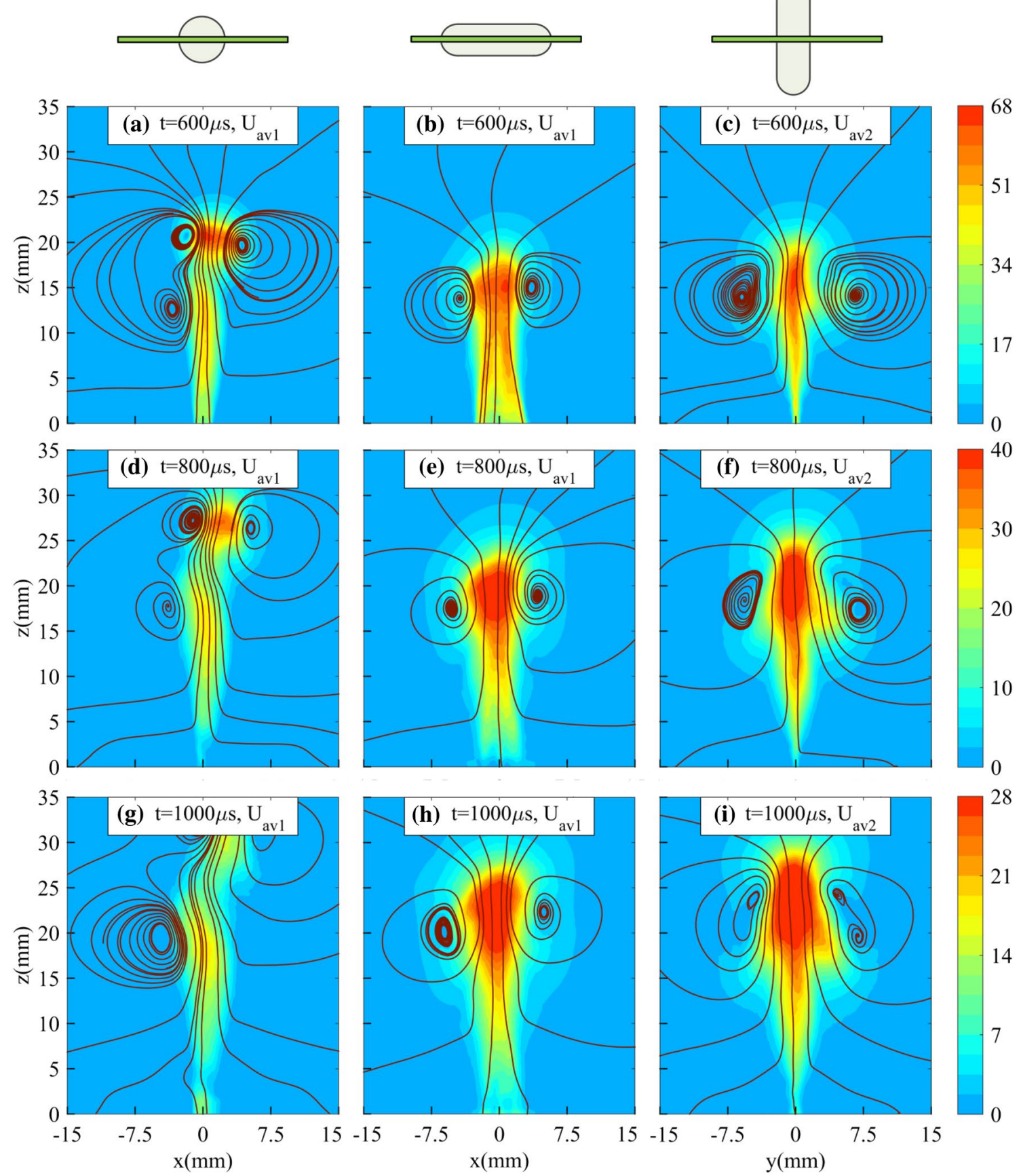

Fig. 13 Phase-lock averaged velocity field between $t=600 \mu \mathrm{s}$ and $t=1000 \mu \mathrm{s}$. Measurement plane and orifice shape are shown in the top line. The solid lines displayed are streamlines

on the phase-averaged flow fields, the time-averaged flow fields $\bar{U}_{i}(x, y, z)(i=x, y, z)$ are calculated as follows,

$$
\begin{aligned}
\bar{U}_{i}(x, y, z) & =\frac{1}{T_{j e t}} \int_{0}^{T_{j e t}} u_{i}(x, y, z, t) d t \\
& \approx \frac{1}{T_{j e t}} \sum_{j=1}^{N} u_{i}(x, y, z, j) \cdot \Delta t_{j}(i=x, y, z),
\end{aligned}
$$

where $T_{j e t}$ is the jet duration time (1.9 ms). $u_{i}(x, y, z, j)$ is the phase-averaged velocity at the $j$ th phase. $\Delta t$ denotes the time step between subsequent phases.

Focusing on the axial component of velocity, only $\bar{U}_{z}(x, y, z)$ is computed. Contours of are displayed in Fig. 18 as function of the two orifice shapes. The jet boundaries indicated by dash-dot lines are determined by $50 \%$ of the peak axial velocity at different $z$ positions. For the jet 
Fig. 14 Contour surface of $U_{a v 2} \cdot \mathbf{a} t=300 \mu \mathrm{s}, U_{a v 2}=$ $50 \mathrm{~m} / \mathrm{s} ; \mathbf{b} t=400 \mu \mathrm{s}, U_{a v 2}=$ $50 \mathrm{~m} / \mathrm{s} ; \mathbf{c} t=600 \mu \mathrm{s}, U_{a v 2}=$ $50 \mathrm{~m} / \mathrm{s} ; \mathbf{d} t=800 \mu \mathrm{s}, U_{a v 2}=$ $40 \mathrm{~m} / \mathrm{s}$
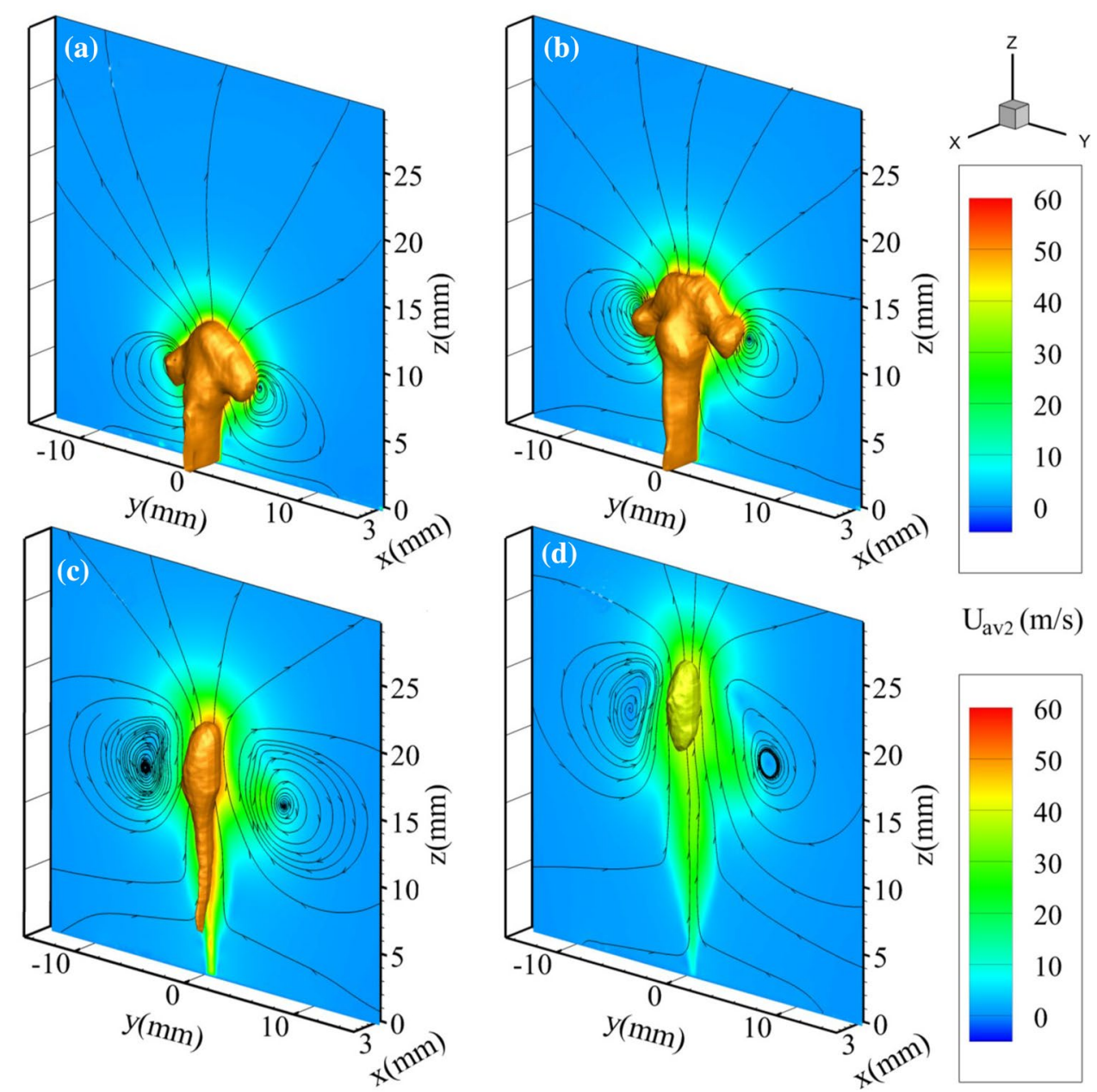

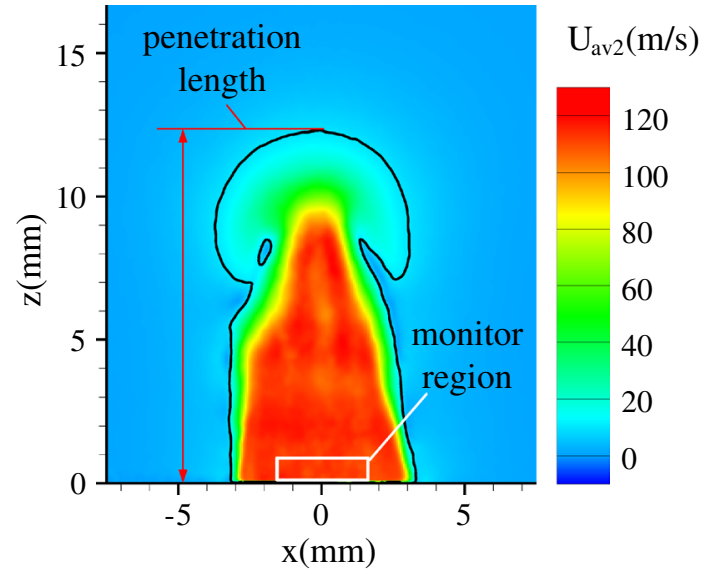

Fig. 15 Penetration length and monitor region

emitted from the round orifice, the time-averaged flow field is similar to that of the steady jets, where a quasi-linear spreading of the jet profile is observed (Hussein et al. 1994) In the case of the slot orifice, the jet profile in $x z$ plane first contracts and then expands. In contrast, the jet profile in $y z$ plane expands monotonically.

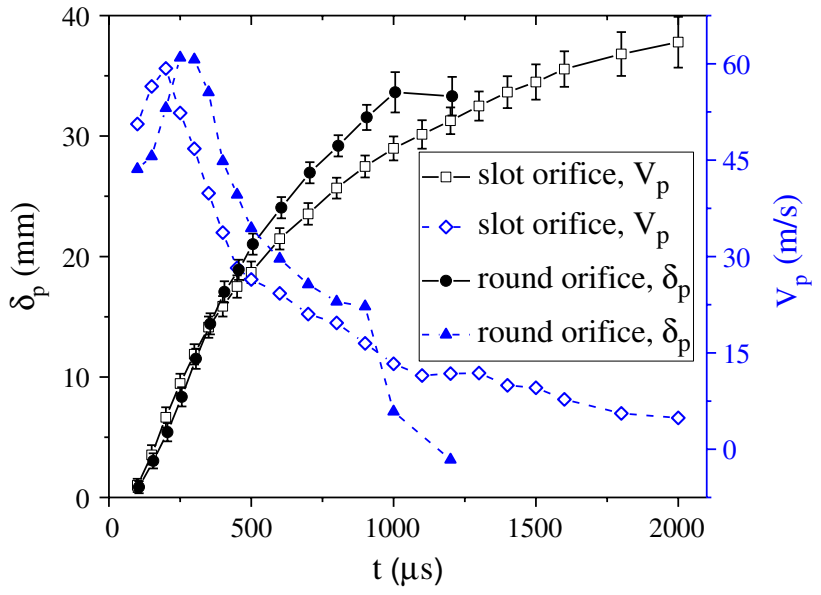

Fig. 16 Variation of penetration length

To quantitatively compare the influence of the orifice shape, the peak jet velocity values at different $z$ positions (denoted as $\bar{U}_{z}(z)$ ) are extracted, as shown in Fig. 19a. Maximum values of $\bar{U}_{z}$ for the two tested cases are almost the same, approximately $27 \mathrm{~m} / \mathrm{s}$. However, the decay rate of 
Fig. 17 Variation of exit velocity for the two tested actuators
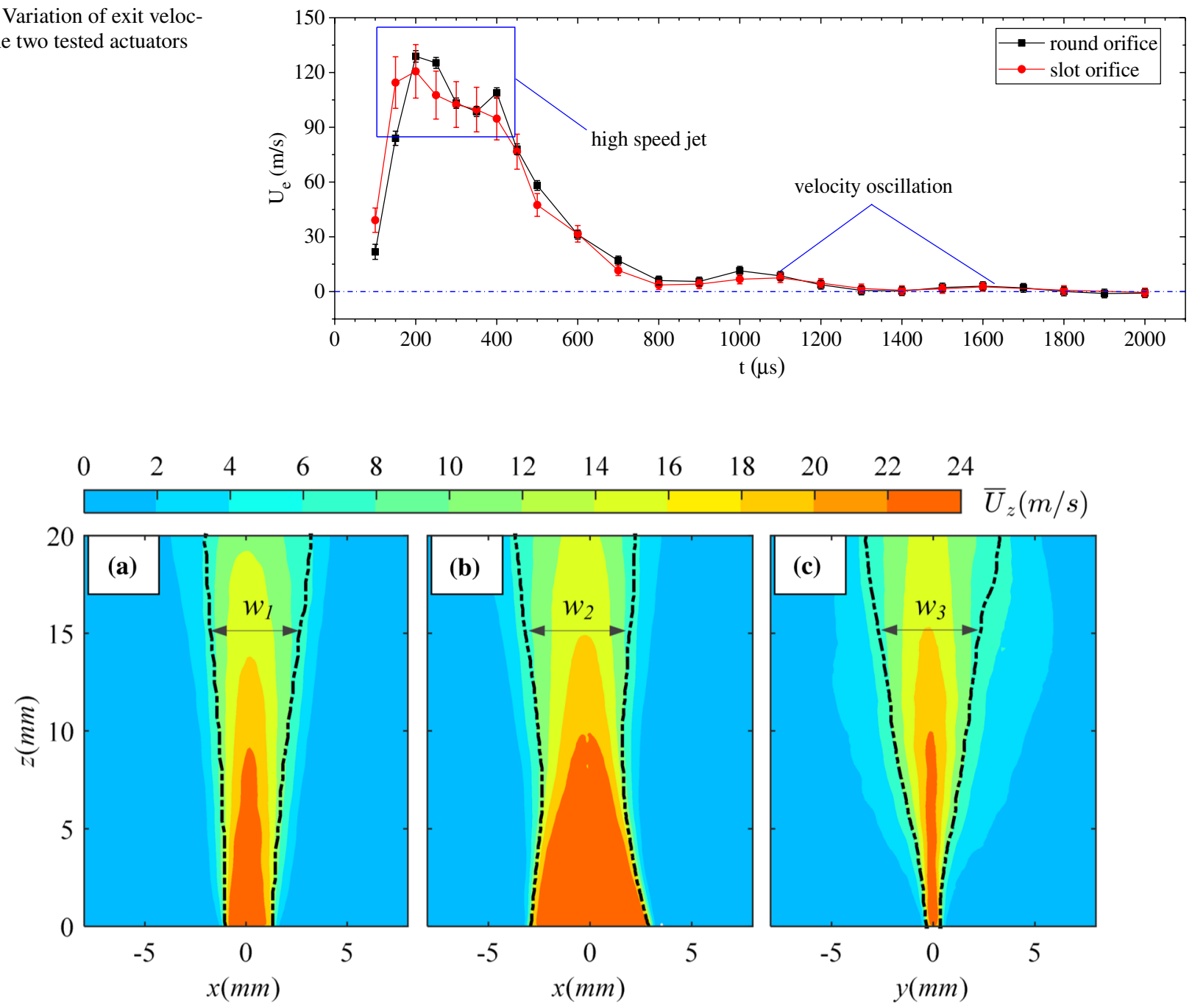

Fig. 18 Contour of time-averaged vertical jet velocity for different orifices. Dash-dot lines indicate the jet boundary determined by $50 \%$ of the peak axial velocity at different $z$ coordinates. Measurement plane and orifice shape for each column are consistent with that in Fig. 13

$\bar{U}_{z}$ for the round orifice is moderately higher than the slot orifice, which is related to the fast propagation of jet body shown in Fig. 16.

The cross-sectional area of the jet body $[S(z)]$ is chosen to characterise the jet spreading rate. To compute $S(z)$, width of the jet body at different $z$ coordinates is first calculated based on the extracted jet boundaries, as illustrated in Fig. 18. For the round orifice, $S(z)$ is defined as the square of the jet width in the $x z$ measurement plane, $w_{1}{ }^{2}(z)$. Whereas for the slot orifice, $S(z)$ is approximated by the product of the two jet width values determined in both symmetry planes, $w_{2}(z) \cdot w_{3}(z)$. Subsequently, $S(z)$ is normalised by the cross-sectional area of the jet at the exit orifice $S(0)$, resulting in a non-dimensional cross-sectional area $\bar{S}(z)$. Variation of $\bar{S}(z)$ is presented in Fig. 19b. As expected, the jet body expands rapidly due to the entrainment of surrounding fluids. At the same $z$ location, the non-dimensional cross-sectional area for the slot orifice is almost twice as that for the round orifice. This ratio is close to the perimeter ratio of slot orifice to round orifice (1.7), testifying the association between jet entrainment rate and exit orifice perimeter.

\section{Conclusions}

A sequential discharge power supply (trigger dischargecapacitive discharge) is exploited to feed a three-electrode large-volume $\left(>1000 \mathrm{~mm}^{3}\right)$ plasma synthetic jet actuator. Two lids with the same exit area but different orifice shapes (round orifice and slot orifice) are designed, and their performance characteristics are investigated based 

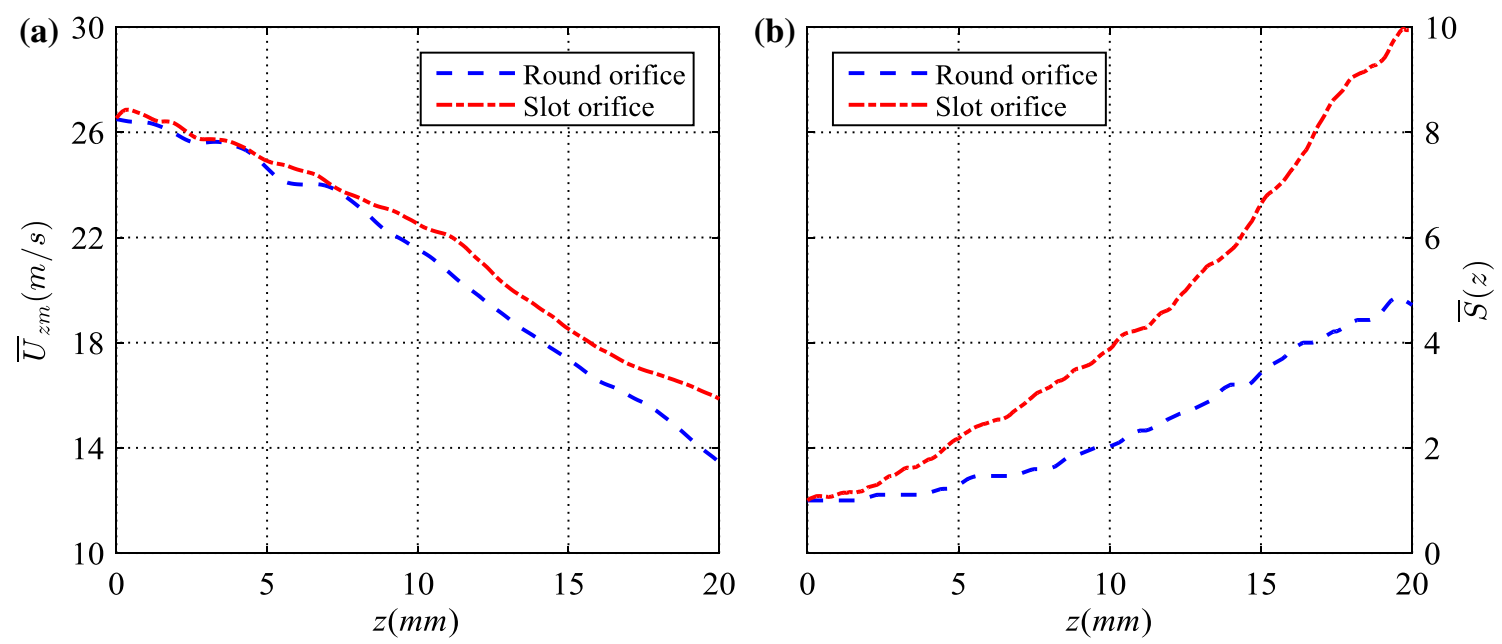

Fig. 19 a Decay of the peak jet velocity at different $z$ coordinates; $\mathbf{b}$ variation of the non-dimensional cross-sectional area of the jet body

on high-speed Schlieren imaging system and phaselocked PIV system.

The shapes of the front vortex rings are consistent with the shapes of the respective exit orifices, since the front vortex ring is rolled from the jet shear layer. For the slot orifice, the elongated vortex ring gradually opens up, and its two ends become severely warped during the propagation. This transformation results from the difference in entrainment rate and propagation velocity. The quasi2D slot jet produces a shear layer in the flow field. The periodical disturbance on the jet exit angle results in a $\mathrm{K}-\mathrm{H}$ instability phenomenon whereupon the high-speed jet continuously vacillates to the left and right during the propagation.

For the slot orifice, the entrainment rate of the surrounding low-energy fluids is almost twice as that for the round orifice, thus resulting in a slower propagation of the jet front. In one period, the body of the slot jet evolves from a mushroom shape to a tadpole shape, and finally to an egg shape. The jet head is always surrounded by the front vortex ring. The exit velocity in one period initially shows a rapid increase, then maintains at a relatively high level $(100-130 \mathrm{~m} / \mathrm{s})$, and finally drops with some small-scale oscillations. The oscillation amplitude is less than $10 \mathrm{~m} / \mathrm{s}$, and the oscillation period is approximately $600 \mu \mathrm{s}$. With the deposited energy and exit area kept constant, orifice shapes have little influence on the variation of jet exit velocity.

Open Access This article is distributed under the terms of the Creative Commons Attribution 4.0 International License (http:// creativecommons.org/licenses/by/4.0/), which permits unrestricted use, distribution, and reproduction in any medium, provided you give appropriate credit to the original author(s) and the source, provide a link to the Creative Commons license, and indicate if changes were made.

\section{References}

Anderson K, Knight D (2012) Plasma jet for flight control. AIAA J 50(9):1855-1873

Belinger A, Hardy P, Barricau P et al (2011) Influence of the energy dissipation rate in the discharge of a plasma synthetic jet actuator. J Phys D Appl Phys 44:365201

Belinger A, Naude N, Cambronne JP et al (2014) Plasma synthetic jet actuator: electrical and optical analysis of the discharge. J Phys D Appl Phys 47:245202

Bletzinger P, Ganguly BN, Van WD et al (2005) Plasmas in high speed aerodynamics. J Phys D Appl Phys 38(4):R33-R57

Caruana D, Barricau P, Hardy P et al (2009) The "plasma synthetic jet" actuator aero-thermodynamic characterization and first flow control applications. In: 47th AIAA aerospace sciences meeting including the new horizons forum and aerospace exposition, 5-8 January 2009, Orlando, Florida. AIAA 2009-1307. doi:10.2514/6.2009-1307

Caruana D, Rogier F, Dufour G et al (2013) The plasma synthetic jet actuator, physics, modeling and flow control application on separation. ONERA - The French Aerospace Lab, Toulouse, pp 1-13

Cattafesta LN, Sheplak M (2011) Actuators for active flow control. Annu Rev Fluid Mech 43:247-272

Corke TC, Enloe CL, Wilkinson SP (2010) Dielectric barrier discharge plasma actuators for flow control. Annu Rev Fluid Mech 42:505-529

Cybyk BZ, Wilkerson JT, Grossman KR et al (2003) Computational assessment of the sparkjet flow control actuator. In: 33rd AIAA fluid dynamics conference and exhibit, 23-26 June 2003, Orlando, Florida. AIAA 2003-3711. doi:10.2514/6.2003-3711

Dhanak MR, Bernardinis BD (1981) The evolution of an elliptic vortex ring. J Fluid Mech 109:189-216

Dufour G, Hardy P, Quint G et al (2013) Physics and models for plasma synthetic jets. Int J Aerodyn 3:47-70

Emerick T, Ali MY, Foster C et al (2014) Sparkjet characterizations in quiescent and supersonic flow fields. Exp Fluids 55:1858

Forte M, Jolibois J, Pons J et al (2007) Optimization of a dielectric barrier discharge actuator by stationary and non-stationary measurements of the induced flow velocity: application to airflow control. Exp Fluids 43:917-928

Glezer A, Amitay M (2002) Synthetic jets. Annu Rev Fluid Mech 34:503-529 
Golbabaei-Asl M, Knight D, Anderson K et al (2013) SparkJet efficiency. In: 51st AIAA aerospace sciences meeting including the new horizons forum and aerospace exposition 07-10 January 2013, Grapevine, Texas. AIAA 2013-0928. doi:10.2514/6.2013-928

Golbabaei-Asl M, Knight D, Wilkinson S (2015) Novel technique to determine Sparkjet efficiency. AIAA J 53:501-504

Greene BR, Clemens NT, Magari P et al (2015) Control of mean separation in shock boundary layer interaction using pulsed plasma jets. Shock waves 25:495-505

Grinstein FF (1995) Self-induced vortex ring dynamics in subsonic rectangular jets. Phys Fluids 7(10):2519-2521

Grossman KR, Cybyk BZ, VanWie DM (2003) Sparkjet actuators for flow control. In: 41st aerospace sciences meeting and exhibit, 6-9 January 2003, Reno, Nevada. AIAA 2003-57. doi:10.2514/6.2003-57

Gutmark EJ, Ibrahim IM, Murugappan S (2011) Dynamics of single and twin circular jets in cross flow. Exp Fluids 50:653-663

Hardy P, Barricau P, Belinger A et al (2010) Plasma synthetic jet for flow control. In: 40th fluid dynamics conference and exhibit, 28 June-1 July 2010, Chicago, Illinois. AIAA 2010-5103. doi:10.2514/6.2010-5103

Hashiehbaf A, Romano GP (2013) Particle image velocimetry investigation on mixing enhancement of non-circular sharp edge nozzles. Int J Heat Fluid Flow 44:208-221

Ho C, Gutmark E (1987) Vortex induction and mass entrainment in a small-aspect-ratio elliptic jet. J Fluid Mech 179:383-405

Hussein HJ, Capp SP, George WK (1994) Velocity measurements in a high-Reynolds-number, momentum-conserving, axisymmetric, turbulent jet. J Fluid Mech 258:31-75

Krothapalli A, Baganoff D, Karamcheti K (1981) On the mixing of a rectangular jet. J Fluid Mech 107:201-220

Laurendeau F, Chedevergne F, Casalis G (2014) Transient election phase modelling of a plasma synthetic jet actuator. Phys Fluids 26:125101

Laurendeau F, Leon O, Chedevergne F, Casalis G (2015) PIV and electric characterization of a plasma synthetic jet actuator. In: 45th AIAA fluid dynamics conference, 22-26 June 2015, Dallas, TX. AIAA 2015-2465. doi:10.2514/6.2015-2465

Lee BHK (1976) Some measurements of spatial instability waves in a round jet. AIAA J 14(3):348-351

Li Y H, Wu Y, Li J (2012) Review of the investigation on plasma flow control in China. Int J Flow Control 4(1+2):1-17

Narayanaswamy V, Rajia L, Clemens NT (2010) Characterization of a high-frequency pulsed-plasma jet actuator for supersonic flow control. AIAA J 48(2):297-305

Narayanaswamy V, Rajia L, Clemens NT (2012) Control of unsteadiness of a shock wave/turbulent boundary layer interaction by using a pulsed-plasma-jet actuator. Phys Fluids 24:076101
Popkin SH, Taylor TM, Cybyk BZ (2013a) Development and application of the sparkjet actuator for high-speed flow control. Johns Hopkins APL Tech Digest 32(1):404-419

Popkin SH, Cybyk BZ, Land HB et al (2013b) Recent performancebased advances in sparkjet actuator design for supersonic flow applications. In: 51st AIAA aerospace sciences meeting including the new horizons forum and aerospace exposition. 07-10 January 2013, Grapevine, Texas. AIAA 2013-0322. doi:10.2514/6.2013-322

Reedy TM, Kale NV, Dutton JC et al (2013) Experimental characterization of a pulsed plasma jet. AIAA J 51(8):2027-2031

Sciacchitano A, Wieneke B (2016) PIV uncertainty propagation. Meas Sci Technol 27:084006

Shin J (2010) Characteristics of high speed electro-thermal jet activated by pulsed DC discharge. Chin J Aeronaut 23(5):518-522

Utkin YG, Keshav S, Kim J et al (2007) Development and use of localized arc filament plasma actuators for high-speed flow control. J Phys D Appl Phys 40:685-694

Wang J, Li YH, Xing F (2009) Investigation on oblique shock wave control by arc discharge plasma in supersonic airflow. J Appl Phys 106:073307

Wang L, Xia ZX, Luo ZB et al (2014a) Effect of pressure on the performance of plasma synthetic jet actuator. Sci China Phys Mechanics Astronomy 57(12):2309-2315

Wang L, Xia ZX, Luo ZB et al (2014b) Three-electrode plasma synthetic jet actuator for high-speed flow control. AIAA J 52(4):879-882

Xu DA, Shneider MN, Lacoste DA et al (2014) Thermal and hydrodynamic effects of nanosecond discharges in atmospheric pressure air. J Phys D Appl Phys 47:235202

Zhang ZB, Wu Y, Jia M et al (2015) Influence of the discharge location on the performance of a three-electrode plasma synthetic jet actuator. Sens Actuators A 235:71-79

Zhu YF, Wu Y, Jia M et al (2015) Influence of positive slopes on ultrafast heating in an atmospheric nanosecond-pulsed plasma synthetic jet. Plasma Sources Sci Technol 24:015007

Zong HH, Kotsonis M (2016) Characterisation of plasma synthetic jet actuators in quiescent flow. J Phys D Appl Phys 49:335202

Zong HH, Cui W, Wu Y et al (2015a) Influence of capacitor energy on performance of a three-electrode plasma synthetic jet actuator. Sens Actuators A 222:114-121

Zong HH, Wu Y, Li YH et al (2015b) Analytic model and frequency characteristics of plasma synthetic jet actuator. Phys Fluids 27:027105

Zong $\mathrm{HH}, \mathrm{Wu}$ Y, Song $\mathrm{HM}$ et al (2016) Influence of geometric parameters on performance of plasma synthetic jet actuators. J Phys D Appl Phys 49(2):025504 